\title{
An updated theoretical scenario for globular cluster stars
}

\author{
S. Cassisi ${ }^{1,2}$, V. Castellani ${ }^{1,3}$, S. Degl'Innocenti ${ }^{3,4}$, and A. Weiss ${ }^{5}$ \\ 1 Osservatorio Astronomico di Collurania, via Mentore Maggini I-64100 Teramo, Italy \\ 2 Dipartimento di Fisica, Universitá de L'Aquila, via Vetoio, 67010 L'Aquila, Italy \\ 3 Dipartimento di Fisica dell'Universitá di Pisa, piazza Torricelli 2, I-56126 Pisa, Italy \\ 4 Istituto Nazionale di Fisica Nucleare, Sezione di Ferrara, via Paradiso 12, I-44100 Ferrara, Italy \\ 5 Max Plank Institut for Astrophysics, Karl Schwarzschild strasse 1, D-85470 Garching b. Munchen, Germany
}

Received February 27; accepted September 22, 1997

\begin{abstract}
In the first part of this paper we revisit the history of theoretical predictions for HB luminosities in old Population II stellar clusters, starting from the results of "old" evolutionary computations to introduce in various steps all the available "new" physics. We discuss the influence of physical ingredients on selected evolutionary parameters, finally presenting models which incorporate all the most recent updating of the relevant physics. The evolutionary behavior of such models is extensively investigated for selected choices about the cluster metallicity, discussing theoretical predictions concerning both cluster isochrones and the calibration of the parameter $R$ in terms of the original amount of He in stellar matter. One finds that the "new" physics has a relevant influence on both these parameters, moving cluster ages into a much better agreement with current cosmological evaluations. This scenario is implemented by a further set of stellar models where element diffusion is taken into account. The comparison between theoretical scenarios with or without diffusion is presented and discussed. A discussion of current observational constraints in the light of the updated theory closes the paper.
\end{abstract}

Key words: stars: evolution; general; fundamental parameters; horizontal-branch

Since galaxies were born in an already expanding Universe, the age of the Universe appears as a safe upper limit for the age of any star and any stellar cluster. The fact that several determinations of globular cluster ages yielded values larger than the age of the Universe as based on current evaluations of the Hubble constant (see, e.g., Van den Bergh 1994; Tanvir et al. 1995) has stimulated a renewed interest in the theory of globular cluster

Send offprint requests to: V. Castellani, Dipartimento di Fisica Università di Pisa, piazza Torricelli 2, 56126 Pisa, Italy, vittorio@astrte.te.astro.it
Pop. II stars. At the same time, significant improvements in the input physics needed for stellar evolution have been made, such that noticeable changes of the theoretical results could be expected. These improvements initially were motivated by the results of helioseismology, which opened a new window into the interior of the Sun, allowing an extremely accurate determination of the solar structure. The efforts undertaken resulted in a new generation of opacity data (Rogers \& Iglesias 1992; Seaton et al. 1994; Iglesias \& Rogers 1996) and equations of state (Mihalas et al. 1990; Rogers et al. 1996), which led to a much better prediction of solar oscillations and also resolved many long-standing problems in our understanding of pulsating stars. In addition, helioseismology required particle diffusion to be taken into account in solar models (see Bahcall et al. 1995 and references therein).

The new opacities and equation of state, along with improvements in low-temperature opacities (e.g. Alexander \& Ferguson 1994), nuclear cross-sections and neutrino emission rates, have now been applied to low-mass metalpoor stars in order to investigate the above-mentioned age problem. Several investigations (Chaboyer \& Kim 1995; Mazzitelli et al. 1995: MDC; VandenBerg et al. 1996; D'Antona et al. 1997; Salaris et al. 1997: Paper I) have already shown that updated models predict lower cluster ages, thus decreasing the size of the discrepancy, if not resolving it. The new physics still needs to be applied to more massive and more metal-rich stars, although some of it, e.g. opacities, already are in use (Bono et al. 1997a,b) However, the full consequences of all improvements have not yet been evaluated. As an example we mention the evolution and pulsations of Cepheid stars.

In the present paper we are concerned with Pop. II stars only. We have a twofold purpose. Firstly, we present stellar models appropriate for globular cluster studies that include all of the improvements listed above. These models cover the complete relevant mass and metallicity range, and include all evolutionary stages from the zero-age main sequence until the end of the helium-burning phase on 
the horizontal branch. Our calculations therefore provide the most up-to-date set of stellar models applicable to all problems of globular cluster dating. In particular, we show for the first time how particle diffusion influences the evolution of low-mass stars until the end of the horizontalbranch phase.

Secondly, we demonstrate how each of the various steps in improving the input physics influences the models. This is important because of the variety of calculations available in the literature that include some but not all of the new physics. In order to compare these results, it is necessary to be able to translate the differences in physical assumptions into differences in stellar properties. In the first part of this paper we will approach this problem, starting from a suitable set of "old" evolutionary computations and introducing, step by step, the available "new" physics in order to make clear the influence of the new assumptions on selected evolutionary parameters. At the end of Sect. 2, we will finally present our best models which will incorporate the most recent improvements in the relevant physics. However, these models will still be calculated ignoring element diffusion.

In Sect. 3 evolutionary predictions for these best models are investigated for selected choices of the cluster metallicity, presenting theoretical predictions for cluster isochrones. This is repeated in Sect. 4 for a set of stellar models where element diffusion is properly taken into account. The comparison between theoretical scenarios with and without diffusion is presented and discussed. Section 5 deals with a discussion of the influence on the $R$-parameter and the consequences for the inferred original amount of helium in stellar matter. The theoretical uncertainties on $R$ are critically discussed and final conclusion given.

\section{Input physics and population II models}

As a starting point we will assume as a reference frame (step 1) the evolutionary scenario presented by Straniero \& Chieffi (1991) and by Castellani et al. (1991: hereinafter CCP), which covers with a homogeneous set of computations the major evolutionary phases experienced by galactic globular cluster stars. As a relevant point, let us here recall that the above evolutionary scenario appears in excellent agreement with computations based on similar physics given by Sweigart (1987); in particular theoretical predictions concerning the mass of the He core at the $\mathrm{He}$ ignition agree to within few thousandths of solar mass.

The "step 1" column in Table 1 gives details of the relevant physics adopted in those models which now can be improved.

Top to bottom one finds:

- Equation of State (EOS) Str88: Straniero (1988) implemented at the lower temperature with Saha equation.

- Radiative Opacity for H, He mixtures (OPAC). LAOL: Los Alamos Opacity Library (Huebner et al. 1977) implemented at the lower temperature with Cox \& Tabor (1976) opacity tables.

- Radiative Opacity for C, O mixtures (OPAC-CO). LAOL: Los Alamos Opacity Library (Huebner et al. 1977). - He burning rates ( $\alpha$-rates). Fow75: Fowler et al. (1975); Harris et al. (1983); Caughlan et al. (1985).

- Neutrino energy losses (NEU). Mun85: Beaudet et al. (1967); Munakata et al. (1985); Richardson et al. (1982).

The updating of the input physics runs as follows:

- EOS from Str88 to OPAL (Rogers 1994; Rogers et al. 1996), implemented in the temperature-density region not covered by OPAL with Str88, plus Saha EOS in the outer stellar layers. The transition from OPAL to other EOS appears smooth and without discontinuities.

- OPAC and OPAC-CO from LAOL to OPAL (Rogers \& Iglesias 1992; Iglesias \& Rogers 1996),

- $\alpha$-rates from Fow75 to Cau88 (Caughlan \& Fowler 1988) and, finally,

- NEU from Mun85 to Haft94 (Haft et al. 1994) for plasma neutrino production and Itoh et al. (1996) for the other kinds of neutrino energy losses.

Electron screening (Graboske et al. 1973; DeWitt et al. 1973) and electron conductivity (Itoh et al. 1983) have not been subject to relevant improvements since that time. As a matter of fact, numerical experiments performed with our code show that neither improvements in strong electron screening, as given by Itoh et al. (1977) and Itoh et al. (1979), nor the alternative approach to weak and intermediate screening (Mitler 1977) affect the evolutionary phases we are dealing with.

Table 1 gives a list of the various modifications in the input physics together with the corresponding values for selected evolutionary quantities. The upper portion of the table gives the steps in updating the physics inputs, whereas in the lower portion of Table 1 one finds selected results concerning the $\mathrm{H}$ burning phase of a $0.8 M_{\odot}$ model ( $Y=0.23, Z=0.0001$ ) and the He burning phase of the same model but assuming the original mass reduced to $0.7 M_{\odot}$ by mass loss. Top to bottom one finds: the luminosity $\left(\log L^{\mathrm{TO}}\right)$ and the age $\left(t^{\mathrm{TO}}\right)$ of the $0.8 M_{\odot} \mathrm{H}$ burning model at the track Turn Off (TO), the luminosity $\left(\log L^{\text {flash }}\right)$, the age $\left(t^{\text {flash }}\right)$ and the mass $\left(M_{\mathrm{c}}\right)$ of the He core at the He flash and the surface helium abundance $\left(Y_{\mathrm{HB}}\right)$ after the first dredge-up. For the He burning $0.7 M_{\odot}$ one finally finds the Zero Age Horizontal Branch luminosity $\left(\log L_{\mathrm{ZAHB}}\right)$, and effective temperature $\left(\log T_{\text {e ZAHB }}\right)$ together with the time $t_{\mathrm{HB}}$ spent in the central He burning phase as a Horizontal Branch (HB) star. Luminosities and masses are in solar units throughout.

Even a quick inspection of results in Table 1 shows the relevant effects produced by the OPAL-EOS on the MS lifetimes and TO-luminosities, an occurrence already well discussed in the literature (see, e.g., Chaboyer \& Kim 1995). For HB models, one finds that improvements in the opacity of H-rich mixtures have the major effect of moderately increasing the HB luminosity $(\Delta \log L \sim 0.02)$ and decreasing the HB lifetime by $3.4 \%$. As expected, 
Table 1. Steps in the input physics and related selected evolutionary quantities for a $0.8 M_{\odot}, Y=0.23, Z=0.0001$ model, assumed as progenitor of the $0.7 M_{\odot}(Y=0.238) \mathrm{HB}$ model at the bottom of the table. Ages for the $0.8 M_{\odot}$ model and for the $0.7 M_{\odot} \mathrm{HB}$ model are, respectively, in Gyr and in Myr

\begin{tabular}{lcccccccc}
\hline \hline Step & 1 & 2 & 3 & 4 & 5 & 6 & 7 & 8 \\
EOS & Str88 & Str88 & Str88 & OPAL & OPAL & OPAL & OPAL & OPAL \\
OPAC & LAOL & OPAL & OPAL & OPAL & OPAL & OPAL & OPAL & OPAL \\
OPAC-CO & LAOL & LAOL & OPAL & OPAL & OPAL & OPAL & OPAL & OPAL \\
$\alpha$-rates & Fow75 & Fow75 & Fow75 & Fow75 & Cau88 & Cau88 & Cau88 & Cau88 \\
NEU & Mun85 & Mun85 & Mun85 & Mun85 & Mun85 & Haft94 & Mun85 & Haft94 \\
\hline & & & & & & & & \\
$\log L^{\mathrm{TO}}\left(L_{\odot}\right)$ & 0.384 & 0.382 & 0.382 & 0.411 & - & - & 0.410 & 0.410 \\
$t^{\mathrm{TO}}(\mathrm{Gyr})$ & 13.62 & 13.50 & 13.50 & 11.58 & - & - & 11.58 & 11.58 \\
$\log L^{\text {flash }}\left(L_{\odot}\right)$ & 3.245 & 3.279 & 3.279 & 3.275 & - & - & 3.291 & 3.322 \\
$t^{\text {fash }}(\mathrm{Gyr})$ & 15.28 & 15.17 & 15.17 & 13.06 & - & - & 13.22 & 13.22 \\
$M_{\mathrm{c}}\left(M_{\odot}\right)$ & 0.5054 & 0.5098 & 0.5098 & 0.5054 & 0.5054 & 0.5054 & 0.5092 & 0.5152 \\
$Y_{\mathrm{HB}}$ & 0.238 & 0.238 & 0.238 & 0.238 & 0.238 & 0.238 & 0.238 & 0.238 \\
\hline & & & & & & & & \\
$\log L_{\mathrm{ZAHB}}\left(L_{\odot}\right)$ & 1.617 & 1.635 & 1.635 & 1.656 & 1.663 & 1.663 & - & 1.666 \\
$\log T_{\mathrm{e} \text { ZAHB }}(\mathrm{K})$ & 3.976 & 3.991 & 3.990 & 3.983 & 3.974 & 3.974 & - & 4.010 \\
$t_{\text {HB }}(\mathrm{Myr})$ & 96.97 & 93.71 & 87.17 & 87.65 & 80.44 & 80.28 & - & 75.02 \\
\hline \hline
\end{tabular}

CO opacity affects only the advanced phases of central $\mathrm{He}$ burning, decreasing the HB lifetimes by a further $7 \%$. As a whole, one finds that the major effect of the new opacities is the decrease of HB lifetimes by the not negligible amount of about $10 \%$. Step 4 in Table 1 shows that the passing from the previous EOS to the more recent OPAL EOS does not affect $\mathrm{HB}$ lifetimes; however one finds that the HB luminosity increases by a further $\Delta \log L \sim 0.02$, in spite of the of the small decreases in $M_{\mathrm{c}}$, whereas the age of the flashing RG decreases by about 2 Gyr.

Steps 5, 6, 7 and 8 finally report the effect of improved evaluations of the triple $\alpha$ nuclear reactions and of the plasma neutrino energy loss rates. On very general ground, one expects that both these mechanisms affect the He ignition at the flash, affecting in turn the structure of the initial ZAHB models. To disentangle this effect from the effect on the physics of HB models, step 5 and 6 concern only ZAHB models, introducing the new rates for $3 \alpha$ reactions (Caughlan \& Fowler 1988) and for plasma neutrino production (Haft et al. 1994) in two subsequent steps for the fixed value of the ZAHB Helium core mass given by the result of step 4 . One finds that the new $3 \alpha$ rates further increase, though slightly, the HB luminosity, whereas HB lifetimes are again substantially decreased by a further $8 \%$. On the contrary, one finds that HB structures are only marginally affected by the NEU treatment, as predicted earlier (Gross 1973).

Step 7 shows the effect of new $3 \alpha$ rates on $\mathrm{H}$ burning models as HB progenitors. Finally, step 8 gives the results for our "best" models where all the available updating of the physics have been taken into account. Due to the effect of both $3 \alpha$ rates and NEU, the He flash is delayed and the peak luminosity of the RG structures is increased, becom- ing about 0.2 mag brighter than in Straniero \& Chieffi (1991; step 1 in Table 1). Correspondingly the value of $M_{\mathrm{c}}$ "jumps" from $0.5054 M_{\odot}$ to $0.5152 M_{\odot}$, contributing to a further increase of the HB luminosity. From data in Table 1 , one recognizes that $3 \alpha$ rates and NEU give a similar contribution to the quoted increase of $M_{\mathrm{c}}$. As a whole, one finds that passing from CCP to present best models the major modifications concerning $\mathrm{HB}$ evolution are given by the increase of the ZAHB luminosity by about $\Delta \log L \sim 0.06(\sim 0.15 \mathrm{mag})$ and by the decrease of HB lifetimes by the huge amount of, about, $23 \%$. As one can easily understand, and as we will discuss later on, this will have rather dramatic effects on current calibration of the $R$ parameter.

To orientate the reader in the current literature, let us review available theoretical estimates in terms of the quoted physical scenarios. As a starting point, let us notice that CCP computations adopt more or less the same input physics adopted in previous computations (as, e.g., Sweigart 1987; Dorman \& VandenBerg 1989; Lee \& Demarque 1990). Dorman (1992) adopts neutrino energy losses and opacities as in CCP, improving nuclear reactions rates as in Caughlan \& Fowler (1988) but taking the EOS from Eggleton et al. (1973). Dorman et al. (1993) adopt the same inputs as Dorman (1992), but lowtemperature opacities from Alexander (1975). Mazzitelli et al. (1995) have OPAL EOS and opacity, but using Dappen et al. (1988) EOS in H burning models (as stated in D'Antona et al. 1997, who updated the turn off models with OPAL EOS); nuclear reactions rates are from Harris et al. (1983) and neutrinos from Itoh et al. (1989). Salaris et al. (1997) models overlap present step 4 assumptions. As a result, one finds that our step 8 is till now the first 
one including all available updating of the input physics. According to such an evidence, in the following section we will investigate the evolutionary behavior of similar models, discussing the calibration of the most relevant evolutionary parameters.

\section{2. "Best" canonical models}

The evolutionary behavior of our "best" models, as defined by step 8 in the previous section, has been investigated for selected choices on the assumed star metallicity and adopting everywhere an original amount of $\mathrm{He}$ given by $Y=0.23$ as a suitable value for population II stars. In all cases we assumed solar scaled composition as given by Grevesse \& Noels (1993). However, alphaenhanced distributions can be taken into account bearing in mind the scaling law discussed by Salaris et al. (1993). It is worth noting that the validity of such a relation has been recently questioned by VandenBerg \& Irwin (1997), but for a metal-rich regime $([\mathrm{Fe} / \mathrm{H}]>-0.8)$ and for large $\alpha$-enhancement factors $([\alpha / \mathrm{Fe}]>0.3$; see also Weiss et al. 1995 for the same topic), i.e., for values beyond the range suitable for globular cluster stars. Table 2 gives selected

Table 2. Evolutionary properties at the TO for step 8 "best" models. The age at the track TO is in Gyr

\begin{tabular}{ccccc}
\hline \hline$Z$ & $\begin{array}{c}M \\
\left(M_{\odot}\right)\end{array}$ & $\begin{array}{r}t^{\mathrm{TO}} \\
(\mathrm{Gyr})\end{array}$ & $\begin{array}{c}\log L^{\mathrm{TO}} \\
\left(L_{\odot}\right)\end{array}$ & $\begin{array}{c}\log T_{\mathrm{e}}^{\mathrm{TO}} \\
(\mathrm{K})\end{array}$ \\
\hline 0.0001 & 0.8 & 11.6 & 0.410 & 3.826 \\
\hline & & & & \\
0.0002 & 0.6 & 33.3 & -0.021 & 3.773 \\
0.0002 & 0.7 & 18.6 & 0.188 & 3.797 \\
0.0002 & 0.8 & 11.2 & 0.378 & 3.824 \\
0.0002 & 0.9 & 7.4 & 0.572 & 3.859 \\
0.0002 & 1.0 & 5.0 & 0.723 & 3.899 \\
\hline & & & & \\
0.001 & 0.6 & 35.6 & -0.178 & 3.755 \\
0.001 & 0.7 & 20.0 & 0.060 & 3.777 \\
0.001 & 0.8 & 11.7 & 0.231 & 3.799 \\
0.001 & 0.9 & 7.4 & 0.393 & 3.822 \\
0.001 & 1.0 & 5.0 & 0.577 & 3.852 \\
\hline & & & & \\
0.006 & 0.65 & 38.3 & -0.250 & 3.729 \\
0.006 & 0.7 & 29.1 & -0.140 & 3.740 \\
0.006 & 0.8 & 16.8 & 0.017 & 3.759 \\
0.006 & 0.9 & 9.5 & 0.134 & 3.777 \\
0.006 & 1.0 & 5.5 & 0.263 & 3.796 \\
\hline \hline
\end{tabular}

data of the models at the track turn-off (TO) for the various choices for the stellar mass and for metallicities $Z=0.0001,0.0002,0.001$ and 0.006 . Left to right one finds: the metallicity $(Z)$, the mass of the model $(M)$, the age $\left(t^{\mathrm{TO}}\right)$, the luminosity $\left(\log L^{\mathrm{TO}}\right)$ and the effective temperature $\left(\log T_{\mathrm{e}}^{\mathrm{TO}}\right)$ at the track Turn Off (TO).

Table 3. Selected TO quantities for $\mathrm{H}$ burning isochrones for the labeled values of metallicity and age

\begin{tabular}{ccccc}
\hline \hline & & & & \\
& age & $\log L^{\mathrm{TO}}$ & $\log T_{\mathrm{e}}^{\mathrm{TO}}$ & $M^{\mathrm{TO}}$ \\
$(\mathrm{Gyr})$ & $\left(L_{\odot}\right)$ & $(\mathrm{K})$ & $\left(M_{\odot}\right)$ \\
\hline & & & & \\
0.0002 & 8 & 0.595 & 3.853 & 0.877 \\
0.0002 & 9 & 0.547 & 3.843 & 0.859 \\
0.0002 & 10 & 0.500 & 3.835 & 0.834 \\
0.0002 & 11 & 0.457 & 3.827 & 0.812 \\
0.0002 & 12 & 0.393 & 3.821 & 0.790 \\
0.0002 & 13 & 0.357 & 3.817 & 0.773 \\
0.0002 & 14 & 0.329 & 3.813 & 0.758 \\
0.0002 & 15 & 0.303 & 3.809 & 0.744 \\
0.0002 & 16 & 0.278 & 3.805 & 0.731 \\
0.0002 & 17 & 0.255 & 3.802 & 0.719 \\
0.0002 & 18 & 0.234 & 3.799 & 0.709 \\
\hline 0.001 & 8 & 0.455 & 3.820 & 0.897 \\
0.001 & 9 & 0.411 & 3.814 & 0.869 \\
0.001 & 10 & 0.373 & 3.809 & 0.845 \\
0.001 & 11 & 0.339 & 3.804 & 0.824 \\
0.001 & 12 & 0.309 & 3.799 & 0.806 \\
0.001 & 13 & 0.256 & 3.796 & 0.786 \\
0.001 & 14 & 0.231 & 3.793 & 0.772 \\
0.001 & 15 & 0.208 & 3.790 & 0.758 \\
0.001 & 16 & 0.187 & 3.787 & 0.746 \\
0.001 & 17 & 0.167 & 3.784 & 0.734 \\
0.001 & 18 & 0.148 & 3.782 & 0.723 \\
\hline 0.006 & 9.5 & 0.281 & 3.781 & 0.932 \\
0.006 & 10 & 0.262 & 3.779 & 0.921 \\
0.006 & 11 & 0.229 & 3.775 & 0.900 \\
0.006 & 12 & 0.203 & 3.772 & 0.881 \\
0.006 & 13 & 0.167 & 3.769 & 0.862 \\
0.006 & 14 & 0.146 & 3.767 & 0.847 \\
0.006 & 15 & 0.114 & 3.764 & 0.831 \\
0.006 & 16 & 0.096 & 3.762 & 0.819 \\
0.006 & 17 & 0.079 & 3.760 & 0.807 \\
0.006 & 18 & 0.059 & 3.757 & 0.796 \\
\hline \hline & & & &
\end{tabular}

On the basis of these evolutionary tracks $\mathrm{H}$ burning isochrones have been computed for the quoted assumed metallicities and covering the range of ages suitable for galactic globular cluster stars. Table 3 gives detailed information on the isochrone TO luminosity and effective temperature. Left to right one finds: the metallicity $(Z)$, the age $\left(t^{\mathrm{TO}}\right)$, the luminosity $\left(\log L^{\mathrm{TO}}\right)$, the effective temperature $\left(\log T_{\mathrm{e}}^{\mathrm{TO}}\right)$ and the mass of the model $\left(M^{\mathrm{TO}}\right)$ at the isochrone Turn Off (TO). As expected, data for the case $Z=0.0002$ overlap similar computations presented in Paper I, since passing from step 4 to step 8 affects only the advanced evolution of RG and HB structures. 
Thus present computations may be regarded as an extension to larger metallicities of the quoted computations.

We agree with the comment of our unknown referee about the risk of using TO luminosity as a parameter to derive cluster ages. From an observational point of view it appears quite difficult to define this parameter with high accuracy (see, e.g., Richer et al. 1988); the average uncertainty on the TO magnitude can be estimated of the order of $\simeq \pm 0.10 \mathrm{mag}$, which leads to an uncertainty on the derived age of the order of \pm 1.5 Gyr (see Chaboyer et al. 1996 a for a discussion on this point). Moreover, from a theoretical point of view, the TO being defined as the bluest point on the isochrone, the determination of the TO magnitude $M_{v}(\mathrm{TO})$ is related to the isochrone color (Chaboyer 1995; Chaboyer et al. 1996b), thus depending on the stellar effective temperature, i.e., on the stellar radius. This radius can be affected by significant uncertainties, depending on the theoretical treatment of convection in superadiabatic layers (MDC). For such reasons, the use of other age indicators (see, e.g., Chaboyer et al. 1996b) has been suggested in several investigations. However, since such a parameter is still widely in use, let us discuss in some detail our results, to allow a comparison with previous results appeared in the literature.

The best fit of the data for the dependence of the TO luminosity on the cluster ages gives the analytical relations:

$\log t_{9}=-0.946 \log L^{\mathrm{TO}}+1.465(Z=0.0002)$

$\log t_{9}=-1.117 \log L^{\mathrm{TO}}+1.414(Z=0.001)$

$\log t_{9}=-1.239 \log L^{\mathrm{TO}}+1.325(Z=0.006)$

where $t_{9}$ is the cluster age in Gyr. These relations reproduce the computational results in the range 8 to 18 Gyr with a maximum error of a few times $10^{8}$ years. As a result, one finds that, for each given age, the TO luminosities are predicted to decrease with metallicity with a slope in fair agreement with a large body of previous predictions but with lower predicted luminosities for each given age. This is shown in Fig. 1, which compares the dependence of present TO luminosities on the assumed metallicity for a given age $(t=12 \mathrm{Gyr})$ with similar results already appeared in the literature. In the figure (as well as in some other following figures) theoretical expectations for O-enhanced mixtures (Bergbusch \& VandenBerg 1992; VandenBerg 1992; Dorman et al. 1993) are reported in terms of the total fraction of heavy elements.

Figure 1 shows that present results represent in all cases a lower boundary for current evaluations of TO luminosities, thus decreasing current age estimates for each given TO luminosity. More in detail, present results predict TO luminosities systematically lower by about $\Delta \log L \simeq 0.05$ with respect to previous computations, with the exception of Mazzitelli et al. (1995: MDC) who predict again larger luminosities, but with a difference which decreases at the larger metallicities. The equations given above imply that the quoted decrease $\Delta \log L \simeq 0.05$ gives a decrease by about $10 \%$ in previous estimates of globular cluster ages. We will discuss this point further in the final section.

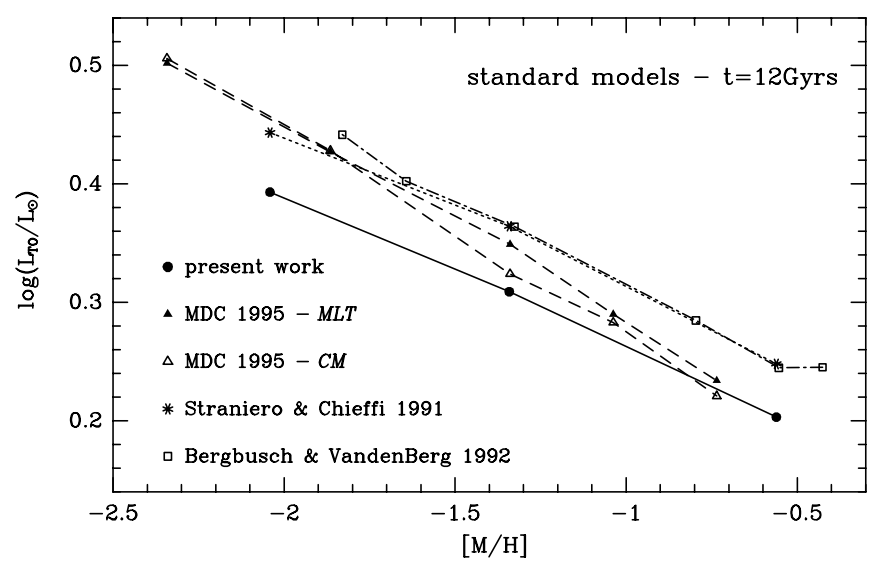

Fig. 1. Behavior of the TO luminosity on the assumed metallicity for a given age $(t=12$ Gyr). Results for present "best" canonical models are compared with similar results available in the literature. For the MDC 1995 models CM indicates the adoption by the authors of the Canuto \& Mazzitelli (1991) treatment of overadiabatic convection while MLT indicates the adoption of the usual mixing length theory

Apart from the problem of TO luminosities, H burning models deserve further attention as progenitors of $\mathrm{He}$ burning models, determining the structural parameters which will constrain the evolutionary behavior and, in particular, the luminosity of HB stars. Computational results

Table 4. Selected evolutionary quantities for $0.8 M_{\odot}$ Red Giant models without diffusion. The age at the He flash is in Gyr

\begin{tabular}{cccccc}
\hline \hline$Z$ & $\begin{array}{c}M_{\mathrm{c}} \\
\left(M_{\odot}\right)\end{array}$ & $Y_{\mathrm{HB}}$ & $\begin{array}{c}t^{\text {flash }} \\
(\mathrm{Gyr})\end{array}$ & $\begin{array}{c}\log L^{\text {flash }} \\
\left(L_{\odot}\right)\end{array}$ & $\begin{array}{c}\log L^{\text {bump }} \\
\left(L_{\odot}\right)\end{array}$ \\
\hline 0.0001 & 0.515 & 0.238 & 13.22 & 3.322 & 2.245 \\
\hline 0.0002 & 0.511 & 0.239 & 13.22 & 3.349 & 2.168 \\
\hline 0.001 & 0.503 & 0.242 & 14.52 & 3.396 & 1.943 \\
\hline 0.006 & 0.494 & 0.246 & 20.89 & 3.442 & 1.535 \\
\hline \hline
\end{tabular}

concerning those parameters are reported in Table 4 for the labeled assumptions about the cluster metallicity. Left to right one finds: the metallicity $(Z)$, the mass $\left(M_{\mathrm{c}}\right)$ of the He core at the He flash, the surface helium abundance $\left(Y_{\mathrm{HB}}\right)$ after the first dredge-up, the age $\left(t^{\text {flash }}\right)$ and the 
luminosity $\left(\log L^{\text {flash }}\right)$ at the He flash and the mean value between the minimum and the maximum in luminosity ( $\left.\log L^{\text {bump }}\right)$ during the RGB "bump". Data in Table 4 will allow the approach of He burning phases adopting self-consistent evolutionary values for the two parameters characterizing a ZAHB structure, namely the mass of the He core $\left(M_{\mathrm{c}}\right)$ and the He abundance in the stellar envelope. Since both values depend only marginally on the assumptions made about the cluster ages, Table 4 reports the values corresponding to a $0.8 M_{\odot}$ evolving Red Giant which can be safely assumed as representative of theoretical expectations in a sufficiently large range of ages. Here let us notice that the discussed increase (see Table 1) of the predicted luminosity of the RG tip would affect the current estimate of the Hubble constant $H_{0}$ when using such a feature as a distance indicator. As a matter of the fact, one easily finds that the quoted increase by $0.2 \mathrm{mag}$ in the top RG luminosity implies an increase by about $10 \%$ in the distance and, in turn, a decrease by the same amount of the $H_{0}$ estimate.

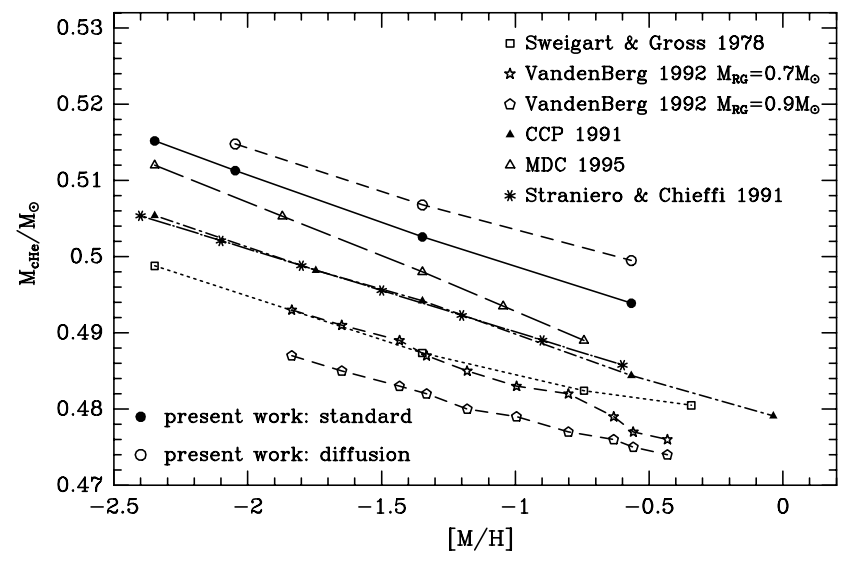

Fig. 2. He core masses at the He flash as a function of metallicity for present models (canonical and with element diffusion) as compared with similar data already appeared in the literature

The amount of extra He $(\Delta Y)$ brought to the stellar surface by the first dredge up appears in good agreement with similar evaluations already given in the literature (see, e.g., Castellani \& Degl'Innocenti 1995 and references therein). Figure 2 compares present masses of the He cores in the flashing Red Giants with previous results. Again one finds that all current evaluations but MDC have a rather similar dependence on the assumed metallicity. However, one finds that our "best" models in all cases predict $M_{\mathrm{c}}$ values larger than previous predictions; this acts in the sense of increasing the expected luminosity of ZAHB structures.

By adopting $M_{\mathrm{c}}$ and $\Delta Y$ values from $\mathrm{H}$ burning models we are now in the position of predicting the evolutionary behavior of He burning Horizontal Branch (HB) structures. Table 5 gives detailed informations on the HR
Table 5. Selected evolutionary quantities for ZAHB models without diffusion. The time spent during the central helium burning phase (in Myr) is compared with results from CCP

\begin{tabular}{|c|c|c|c|c|}
\hline \multicolumn{5}{|c|}{$Z=0.0001 Y_{\mathrm{HB}}=0.238 M_{\mathrm{c}}=0.515 M_{\odot}$} \\
\hline $\begin{array}{c}M \\
\left(M_{\odot}\right)\end{array}$ & $\begin{array}{l}\log L \\
\left(L_{\odot}\right)\end{array}$ & $\begin{array}{c}\log T_{\mathrm{e}} \\
(\mathrm{K})\end{array}$ & $\begin{array}{c}t_{\mathrm{He}} \\
(\mathrm{Myr})\end{array}$ & $\begin{array}{c}t_{\mathrm{He}}^{\mathrm{CCP}} \\
(\mathrm{Myr})\end{array}$ \\
\hline 0.53 & 1.357 & 4.410 & 93.0 & - \\
\hline 0.55 & 1.395 & 4.320 & 88.2 & 113.6 \\
\hline 0.60 & 1.471 & 4.217 & 82.6 & 105.3 \\
\hline 0.65 & 1.582 & 4.108 & 78.5 & 100.6 \\
\hline 0.70 & 1.662 & 4.010 & 75.0 & 96.9 \\
\hline 0.75 & 1.727 & 3.926 & 74.4 & 94.7 \\
\hline 0.77 & 1.744 & 3.900 & 73.9 & - \\
\hline 0.80 & 1.769 & 3.860 & - & 91.3 \\
\hline \multicolumn{5}{|c|}{$Z=0.001 Y_{\mathrm{HB}}=0.242 M_{\mathrm{c}}=0.503 M_{\odot}$} \\
\hline $\begin{array}{c}M \\
\left(M_{\odot}\right)\end{array}$ & $\begin{array}{l}\log L \\
\left(L_{\odot}\right)\end{array}$ & $\begin{array}{c}\log T_{\mathrm{e}} \\
(\mathrm{K})\end{array}$ & $\begin{array}{c}t_{\mathrm{He}} \\
(\mathrm{Myr})\end{array}$ & $\begin{array}{c}t_{\mathrm{He}}^{\mathrm{CCP}} \\
(\mathrm{Myr})\end{array}$ \\
\hline 0.53 & 1.328 & 4.331 & 98.8 & - \\
\hline 0.55 & 1.373 & 4.257 & 94.2 & 119.9 \\
\hline 0.60 & 1.562 & 4.084 & 86.6 & 111.2 \\
\hline 0.65 & 1.688 & 3.855 & 84.0 & 106.8 \\
\hline 0.70 & 1.747 & 3.739 & 82.5 & 104.3 \\
\hline 0.75 & 1.777 & 3.726 & 81.6 & 101.9 \\
\hline 0.80 & 1.794 & 3.722 & - & - \\
\hline
\end{tabular}

diagram location of Zero Age Horizontal Branch (ZAHB) together with a comparison between present and CCP He burning lifetimes. Left to right one finds: the mass $(M)$, the luminosity $(\log L)$ and the effective temperature $\left(\log T_{\mathrm{e}}\right)$ of the zero-age horizontal-branch, ZAHB, model (following CCP we assume as ZAHB structures the models already evolved by $1 \mathrm{Myr})$, the time $\left(t_{\mathrm{He}}\right)$ spent during the central He burning (until the disappearance of the convective core) and the same quantity $\left(t_{\mathrm{He}}^{\mathrm{CCP}}\right)$ but for the CCP models.

As expected on the basis of the exploratory computations given in the first part of this paper, one finds that the expected luminosity of ZAHB models is substantially increased whereas He burning lifetimes in all cases decrease by more than $20 \%$. Figure 3 presents predictions about the luminosity of the ZAHB model in the RR Lyrae instability strip $\left(\log T_{\mathrm{e}}=3.85\right)$ together with previous results. One finds that "old" computations, as given by CCP or Lee \& Demarque (1990) agree in predicting lower luminosities, by about $\Delta \log L \approx 0.05$. This implies that, when using ZAHB models as "standard candles" to constrain the cluster distance modulus (DM), "old" computations would produce smaller DM, thus lower luminosities of the 


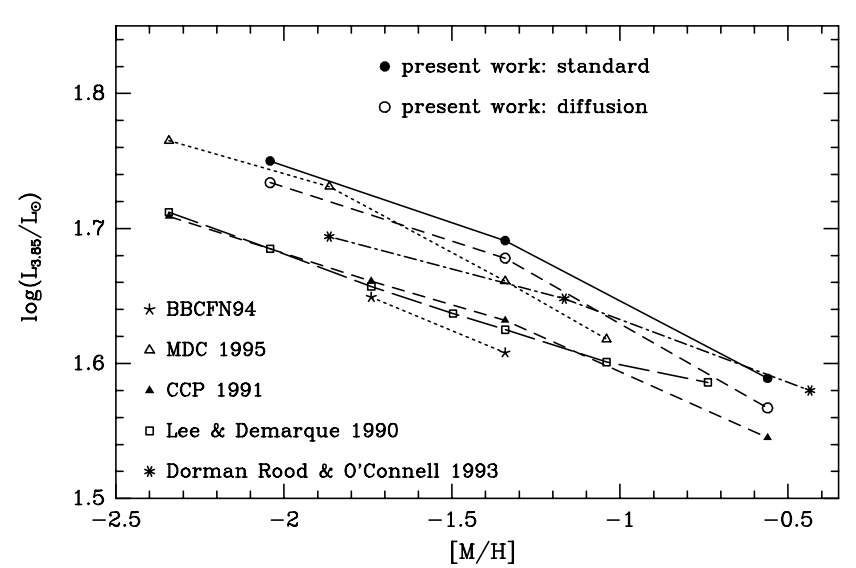

Fig. 3. The ZAHB luminosity at $\log T_{\mathrm{e}}=3.85$, as a function of metallicity for present models, compared with previous results, as labeled $($ BCFN94 = Bertelli et al. 1994)

observed TO and, finally, greater ages. The same figure shows that all the most recent computations agree in predicting more luminous ZAHBs. In particular one finds that at the lowest metallicity, we predict luminosities in close agreement with MDC, notwithstanding the (small) difference in the He core masses. Note that the difference at the larger metallicities can be understood in terms of the different slope of the $M_{\mathrm{c}}$-metallicity relation already shown in Fig. 2.

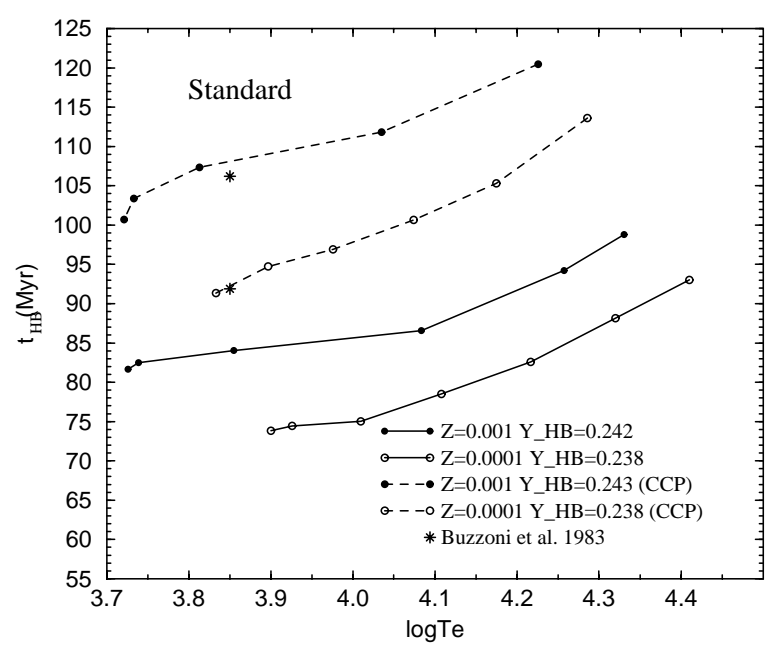

Fig. 4. Central He-burning lifetimes as a function of the ZAHB effective temperature for present models (solid line) compared with similar data in CCP (dashed line) and with the predictions by Buzzoni et al. (1983) for HB models with $\log T_{\mathrm{e}}=3.83$ (stars). Metallicities as labeled

Figure 4 finally compares present He-burning lifetimes with the ones given in CCP and with the value originally predicted by Buzzoni et al. (1983) for the two assumed metallicities. The emerging scenario concerning current evaluation of the amount of original He in globular cluster stars will be discussed in Sect. 5 .

\section{Element diffusion}

A general discussion on the relevance of element diffusion in the evolution of Pop.II stars has been already given in a previous paper (Castellani et al. 1997: Paper II) and it will be not repeated here. Here we only recall that the diffusion of both He and heavy elements is taken into account, according to the algorithm adopted in Thoul et al. (1994). Table 6 gives selected evolutionary properties of

Table 6. Selected evolutionary quantities for models with diffusion at the track TO. The age at the track TO is in Gyr

\begin{tabular}{clrrc}
\hline \hline$Z$ & $\begin{array}{c}M \\
\left(M_{\odot}\right)\end{array}$ & $\begin{array}{c}\mathrm{age}^{\mathrm{TO}} \\
(\mathrm{Gyr})\end{array}$ & $\begin{array}{c}\log L^{\mathrm{TO}} \\
\left(L_{\odot}\right)\end{array}$ & $\begin{array}{c}\log T_{\mathrm{e}}^{\mathrm{TO}} \\
(\mathrm{K})\end{array}$ \\
\hline 0.0002 & 0.6 & 30.8 & -0.078 & 3.751 \\
0.0002 & 0.7 & 17.7 & 0.140 & 3.786 \\
0.0002 & 0.8 & 10.9 & 0.330 & 3.814 \\
0.0002 & 0.9 & 7.1 & 0.536 & 3.848 \\
0.0002 & 1.0 & 4.9 & 0.702 & 3.890 \\
\hline 0.001 & 0.7 & 18.7 & 0.026 & 3.770 \\
0.001 & 0.8 & 10.9 & 0.187 & 3.794 \\
0.001 & 0.9 & 6.7 & 0.348 & 3.818 \\
0.001 & 1.0 & 4.8 & 0.551 & 3.847 \\
\hline 0.006 & 0.65 & 35.1 & -0.255 & 3.721 \\
0.006 & 0.70 & 26.6 & -0.172 & 3.733 \\
0.006 & 0.80 & 15.4 & -0.027 & 3.754 \\
0.006 & 0.90 & 8.7 & 0.101 & 3.774 \\
0.006 & 1.0 & 5.1 & 0.245 & 3.794 \\
\hline \hline
\end{tabular}

models for the various choices on the stellar mass and for the investigated metallicities. As a whole, our results closely follow the general trends discussed by Proffitt \& VandenBerg (1991) in their pioneering paper to which we refer the reader. Figure 5 compares the run in the HR diagram of isochrones with and without element diffusion for a selected metallicity and for the labeled choices on the cluster age whereas Table 7 gives detailed information on the isochrone TO luminosity and effective temperature. Tables 6 and 7 correspond to Tables 2 and 3 given in the previous section for computations neglecting element diffusion. Figure 6 shows the dependence of (isochrones) TO luminosity on cluster age, as compared with similar results but without allowing for element sedimentation.

In the case of sedimentation the best fitting of the data connecting ages to TO luminosities gives the following relation for ages between 8 and $18 \mathrm{Gyr}$ :

$\log t_{9}=-0.924 \log L^{\mathrm{TO}}+1.414(Z=0.0002)$

$\log t_{9}=-1.130 \log L^{\mathrm{TO}}+1.384(Z=0.001)$

$\log t_{9}=-1.170 \log L^{\mathrm{TO}}+1.288(Z=0.006)$ 
Table 7. Selected TO quantities for $\mathrm{H}$ burning isochrones with diffusion for the labeled assumptions about age and original chemical composition

\begin{tabular}{|c|c|c|c|}
\hline \multicolumn{4}{|c|}{$Z=0.0002 Y=0.23$} \\
\hline $\begin{array}{c}\text { age } \\
(\mathrm{Gyr}) \\
\end{array}$ & $\begin{array}{c}\log L^{\mathrm{TO}} \\
\left(L_{\odot}\right)\end{array}$ & $\begin{array}{c}\log T_{\mathrm{e}}^{\mathrm{TO}} \\
(\mathrm{K})\end{array}$ & $\begin{array}{l}M^{\mathrm{TO}} \\
\left(M_{\odot}\right)\end{array}$ \\
\hline 8 & 0.557 & 3.840 & 0.881 \\
\hline 9 & 0.501 & 3.831 & 0.852 \\
\hline 10 & 0.450 & 3.822 & 0.826 \\
\hline 11 & 0.406 & 3.814 & 0.804 \\
\hline 12 & 0.346 & 3.809 & 0.783 \\
\hline 13 & 0.313 & 3.804 & 0.766 \\
\hline 14 & 0.283 & 3.800 & 0.750 \\
\hline 15 & 0.255 & 3.796 & 0.736 \\
\hline 16 & 0.228 & 3.793 & 0.723 \\
\hline 17 & 0.204 & 3.789 & 0.711 \\
\hline 18 & 0.180 & 3.786 & 0.700 \\
\hline \multicolumn{4}{|c|}{$Z=0.001 Y=0.23$} \\
\hline 8 & 0.429 & 3.814 & 0.888 \\
\hline 9 & 0.383 & 3.807 & 0.861 \\
\hline 10 & 0.342 & 3.801 & 0.837 \\
\hline 11 & 0.305 & 3.796 & 0.816 \\
\hline 12 & 0.259 & 3.791 & 0.795 \\
\hline 13 & 0.232 & 3.788 & 0.779 \\
\hline 14 & 0.206 & 3.784 & 0.764 \\
\hline 15 & 0.183 & 3.781 & 0.750 \\
\hline 16 & 0.160 & 3.778 & 0.737 \\
\hline 17 & 0.140 & 3.775 & 0.726 \\
\hline 18 & 0.120 & 3.773 & 0.715 \\
\hline \multicolumn{4}{|c|}{$Z=0.006 Y=0.23$} \\
\hline 9.5 & 0.265 & 3.776 & 0.926 \\
\hline 10 & 0.244 & 3.774 & 0.914 \\
\hline 11 & 0.204 & 3.770 & 0.891 \\
\hline 12 & 0.185 & 3.767 & 0.874 \\
\hline 13 & 0.156 & 3.763 & 0.856 \\
\hline 14 & 0.129 & 3.760 & 0.840 \\
\hline 15 & 0.090 & 3.757 & 0.823 \\
\hline 16 & 0.067 & 3.755 & 0.809 \\
\hline 17 & 0.047 & 3.752 & 0.797 \\
\hline 18 & 0.031 & 3.750 & 0.785 \\
\hline
\end{tabular}

which again reproduce the results to within a few $10^{8}$ years.

According to the discussion given in Paper II for the case $Z=0.0004$, one finds that element diffusion moderately decreases theoretical expectations for TO luminosities for each given cluster age. Figure 6 now shows that such an effect depends on the assumed metallicity, increasing when the metallicity is decreased. As a matter of the fact, the effect of diffusion on the TO luminosity is larger in the most metal poor isochrones since the diffusion in the stellar envelopes is larger due to the thinner convective envelopes on MS stars. At the lowest metallic-

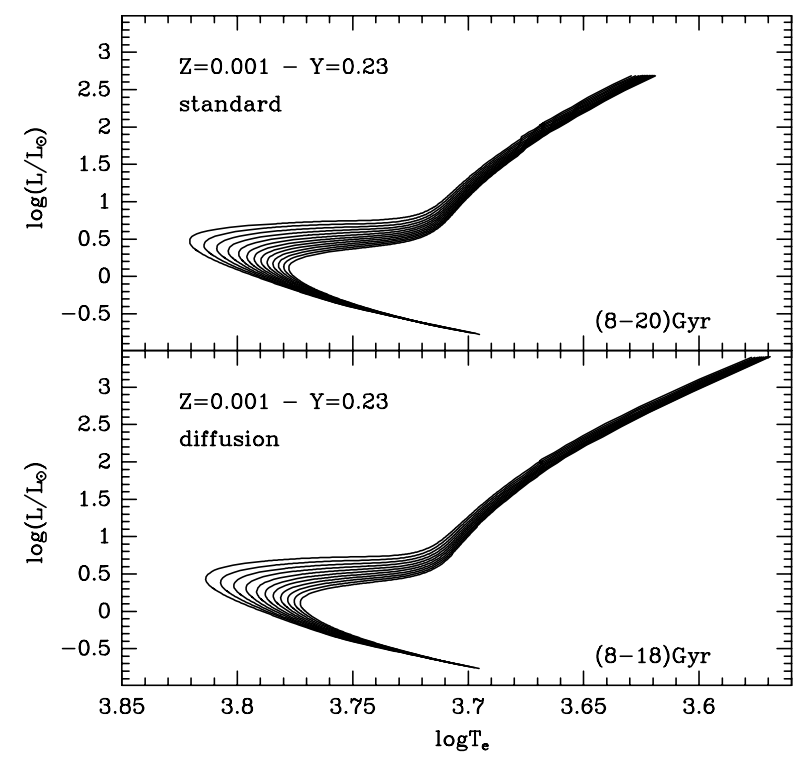

Fig. 5. $\mathrm{H}$ burning isochrones for $Y=0.23, Z=0.001$ and for the labeled ages for the present models without and with element diffusion (upper and lower panel, respectively). The time interval between consecutive isochrones is 1 Gyr. Note that the standard isochrones are calculated until a luminosity lower than that of the helium flash

ity $(Z=0.0001) \Delta \log L \approx 0.04$ : thus, for a given observed TO luminosity, allowing for sedimentation would decrease age estimates by about $10 \%$. On the contrary, when $Z=0.006$ one expects negligible variations.

Table 8 gives selected structural parameters of models at the He flash, to be used as input to the He-burning models (for a discussion of the effects of diffusion on the luminosity of the RGB "bump", see also Cassisi et al. 1997). On this basis we present in Fig. 7 a comparison between HB evolution with or without diffusion; in the same figure the path in the HR diagram of these models is also compared with similar results from CCP. Table 9 gives details on the ZAHB structures and on the corresponding helium burning evolutionary times. HB structures presented in both Fig. 7 and Table 9 assume a $0.8 M_{\odot}$ model as $\mathrm{H}-$ burning progenitor. However, Table 8 shows that, e.g., increasing the RG masses (thus decreasing the cluster age) $M_{\mathrm{c}}$ decreases but $Y_{\mathrm{HB}}$ increases, with balancing effects on the predicted HB luminosities. As a result, numerical experiments disclose that HB data based on a $0.8 M_{\odot}$ progenitor can be safely taken as representative of HB models in the range $0.7<M / M_{\odot}<0.9$, at least, thus covering quite a large range of cluster ages.

Figure 8 summarizes the results of this paper concerning cluster ages, showing the run with metallicities of ZAHB and TO luminosities, with or without diffusion, and for selected choices about the assumed ages. In this figure, data concerning the TO luminosity have been supplemented with similar data but for $Z=0.0004$, as given in Paper II with a "step 4" physics which is fully 


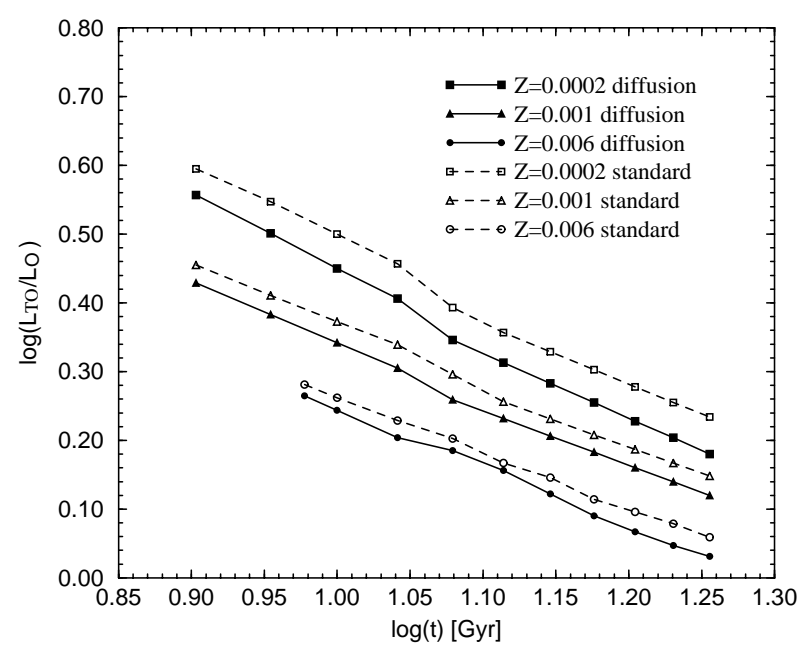

Fig. 6. Dependence of the TO luminosity on the cluster ages for the three labeled metallicities. The results for canonical models (dashed line) are compared with similar results but for models with element sedimentation (solid line)

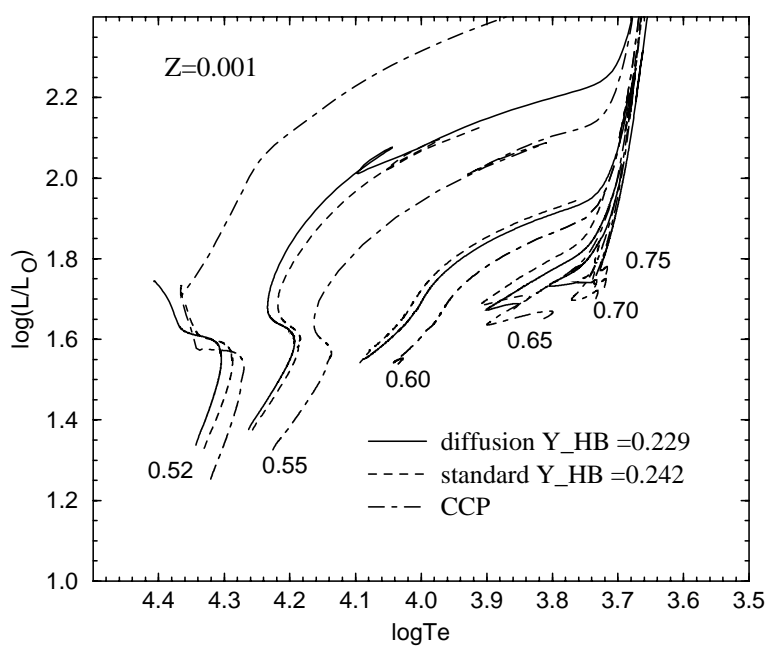

Fig. 7. Comparison of the HB evolution for models with (solid line) and without (dashed line) diffusion for $Z=0.001$ and $Y=0.23$. Similar results from CCP (dot-dashed line) are also shown

compatible with present computation as far as central $\mathrm{H}$ burning models are concerned. As already discussed, present HB are brighter than estimated in Paper II (see Table 1). However, the same Fig. 8 shows that present computations keep predicting that diffusion decreases the HB luminosity by about $\Delta \log L \sim 0.02$, in agreement with the results of Paper II.

Figure 9 shows the calibration of age in terms of the difference in luminosities between ZAHB (taken at $\left.\log T_{\mathrm{e}}=3.85\right)$ and TO, as predicted with or without diffusion, and as compared with original predictions in CCP. As discussed in Paper II one finds that diffusion plays a minor role in that calibration. However, the same Fig. 9

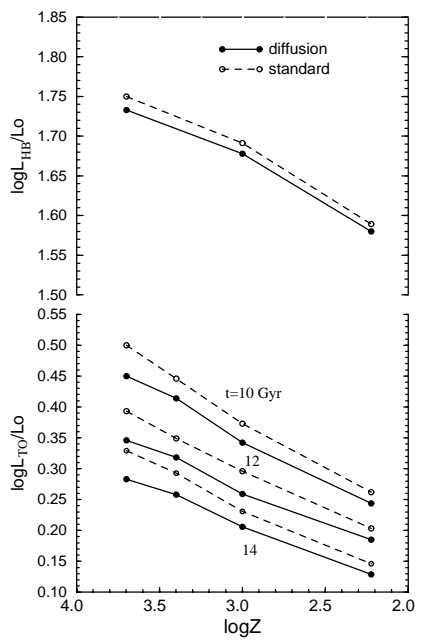

Fig. 8. TO luminosities for selected labeled ages and ZAHB luminosities at $\log T_{\mathrm{e}}=3.85$ as a function of metallicity, for models with (solid line) and without (dashed line) element diffusion

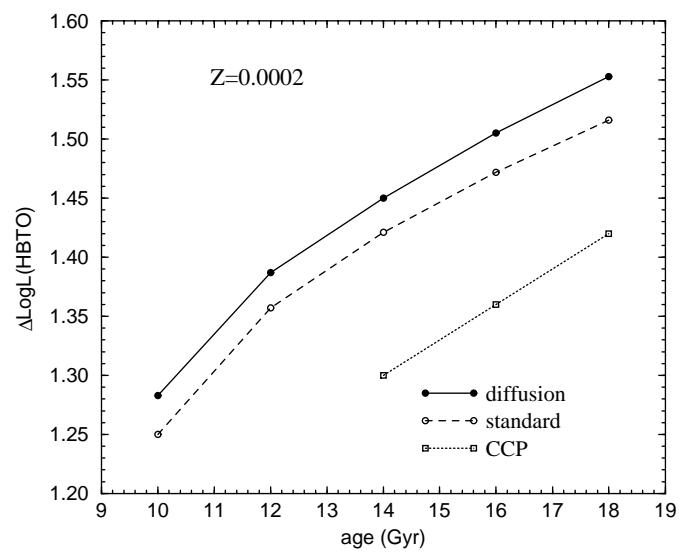

Fig. 9. The calibration of age in terms of the difference in luminosities $(\Delta \log L(\mathrm{HB}-\mathrm{TO}))$ between ZAHB $\left(\right.$ at $\left.\log T_{\mathrm{e}}=3.85\right)$ and TO, as predicted by present models with (solid line) and without diffusion (dashed line) and $Z=0.0002$. The results are compared with original predictions by CCP (dotted line)

shows that the new physics, as a whole, reduces by about 4 Gyr theoretical calibrations based on the old physics.

The detailed comparison between theory and observation is a delicate question, beyond the scope of this theoretical paper. However, one may test present predictions by comparing with recent estimates of HB luminosities derived by recent Hipparcos parallaxes measurements. This is shown in Fig. 10, which compares the data presented by De Boer et al. (1997) with our theoretical predictions converted in $M_{v}, B-V$ magnitudes according to model atmospheres by Kurucz (1992). One finds that the HB luminosity level appears in quite good agreement with the quoted observations. Here we only notice that the two stars which lie below the ZAHB around $B-V \simeq 0$ both have been 
Table 8. Selected evolutionary quantities for stellar models with element diffusion at the He flash. The age at the He flash is in Gyr

\begin{tabular}{|c|c|c|c|c|c|}
\hline$M$ & $M_{\mathrm{c}}$ & $Y_{\mathrm{HB}}$ & $t^{\text {flash }}$ & $\log L^{\text {flash }}$ & $\log L^{\text {bump }}$ \\
\hline \multicolumn{6}{|c|}{$Z=0.0002$} \\
\hline 0.7 & 0.5188 & 0.2144 & 20.33 & 3.359 & 2.056 \\
\hline 0.8 & 0.5148 & 0.2261 & 12.77 & 3.352 & 2.139 \\
\hline 0.9 & 0.5113 & 0.2344 & 8.51 & 3.344 & 2.209 \\
\hline 1.0 & 0.5081 & 0.2402 & 5.96 & 3.334 & 2.290 \\
\hline \multicolumn{6}{|c|}{$Z=0.001$} \\
\hline 0.7 & 0.5107 & 0.2178 & 22.24 & 3.410 & 1.825 \\
\hline 0.8 & 0.5068 & 0.2294 & 13.98 & 3.404 & 1.917 \\
\hline 0.9 & 0.5041 & 0.2377 & 9.28 & 3.398 & 1.983 \\
\hline 1.0 & 0.5018 & 0.2434 & 6.46 & 3.393 & 2.043 \\
\hline \multicolumn{6}{|c|}{$Z=0.006$} \\
\hline 0.7 & 0.5034 & 0.2184 & 31.36 & 3.460 & 1.399 \\
\hline 0.8 & 0.5001 & 0.2308 & 19.91 & 3.457 & 1.495 \\
\hline 0.9 & 0.4975 & 0.2392 & 13.30 & 3.457 & 1.578 \\
\hline 1.0 & 0.4958 & 0.2446 & 9.20 & 3.530 & 1.657 \\
\hline
\end{tabular}

corrected for a rather large reddening $(E(B-V)=0.10)$, one -to our knowledge- devoid of recent metallicity estimates.

As for the outcome of the improved theoretical scenario, let us recall that in Paper I it has been already shown that in the step 4 scenario the color magnitude diagram of a typical metal poor galactic globular can be reproduced by a 12 Gyr (no diffusion) isochrone. Taking into account that, at $\log T_{\mathrm{e}}=3.85$, our best $\mathrm{HB}$ models without diffusion turn out to be more luminous by $\Delta \log L_{\mathrm{ZAHB}} \approx 0.02$, one estimates that the "new" age shifts toward 11 Gyr without sedimentation, and even lower if sedimentation is taken into account.

Figure 11 shows that present results give a rather low dependence of $\Delta \log L(\mathrm{HB}-\mathrm{TO})$ on the cluster metallicity. As a matter of the fact, assuming, e.g. an age of $12 \mathrm{Gyr}$ one finds that passing from $Z=0.0002([\mathrm{Fe} / \mathrm{H}]=-1.97)$ to $Z=0.001([\mathrm{Fe} / \mathrm{H}]=-1.27)$ we predict an increase in $\Delta \log L(\mathrm{HB}-\mathrm{TO})$ corresponding to $\approx 0.08 \mathrm{mag}$, independent of any assumptions about the efficiency of sedimentation; this difference increases when the cluster age is decreased.

\section{The parameter $R$}

Since the pioneering paper by Iben (1968) it is known that evolutionary predictions on the evolution of Pop. II
Table 9. Selected evolutionary quantities for HB models with diffusion. The time spent during the central He burning phase is in Myr

\begin{tabular}{|c|c|c|c|}
\hline \multicolumn{4}{|c|}{$Z=0.0002 Y_{\mathrm{HB}}=0.226 M_{\mathrm{c}}=0.515 M_{\odot}$} \\
\hline $\begin{array}{c}M \\
\left(M_{\odot}\right) \\
\end{array}$ & $\begin{array}{c}\log L \\
\left(L_{\odot}\right) \\
\end{array}$ & $\begin{array}{c}\log T_{\mathrm{e}} \\
(\mathrm{K}) \\
\end{array}$ & $\begin{array}{c}t_{\mathrm{He}} \\
(\mathrm{Myr})\end{array}$ \\
\hline 0.53 & 1.352 & 4.395 & - \\
\hline 0.54 & 1.369 & 4.352 & - \\
\hline 0.55 & 1.386 & 4.318 & 86.6 \\
\hline 0.58 & 1.437 & 4.237 & - \\
\hline 0.60 & 1.478 & 4.192 & 78.1 \\
\hline 0.62 & 1.524 & 4.145 & 78.0 \\
\hline 0.65 & 1.593 & 4.072 & 76.0 \\
\hline 0.70 & 1.678 & 3.952 & 73.9 \\
\hline 0.72 & 1.703 & 3.909 & - \\
\hline 0.75 & 1.734 & 3.849 & 72.9 \\
\hline 0.77 & 1.751 & 3.813 & - \\
\hline 0.80 & 1.773 & 3.772 & 66.6 \\
\hline \multicolumn{4}{|c|}{$Z=0.001 Y_{\mathrm{HB}}=0.229 M_{\mathrm{c}}=0.507 M_{\odot}$} \\
\hline $\begin{array}{c}M \\
\left(M_{\odot}\right) \\
\end{array}$ & $\begin{array}{l}\log L \\
\left(L_{\odot}\right) \\
\end{array}$ & $\begin{array}{c}\log T_{\mathrm{e}} \\
(\mathrm{K}) \\
\end{array}$ & $\begin{array}{c}t_{\mathrm{He}} \\
(\mathrm{Myr}) \\
\end{array}$ \\
\hline 0.53 & 1.337 & 4.343 & 95.0 \\
\hline 0.55 & 1.376 & 4.263 & 92.8 \\
\hline 0.60 & 1.540 & 4.095 & 84.8 \\
\hline 0.65 & 1.671 & 3.866 & 81.4 \\
\hline 0.70 & 1.730 & 3.735 & 80.3 \\
\hline 0.75 & 1.760 & 3.719 & 78.7 \\
\hline
\end{tabular}

stars can be used to constrain the amount of original He in globular cluster stars. Calibrations of the $R$ parameter, i.e., the number ratio between $\mathrm{HB}$ stars and RG more luminous than the $H B$ luminosity level have been given by Buzzoni et al. (1983) and, more recently, by Caputo et al. (1987) and by Bono et al. (1995). According to current estimates, observational values for $R$ appear to range around $R \sim 1.1$. In terms of the quoted calibrations this implies $Y \sim 0.23$, which consequently is the value currently adopted in discussing globular cluster stars.

However, the evolutionary results discussed in the previous sections deeply affect such a scenario. We already found that the updated physics moderately increases theoretical expectations for HB luminosities, largely decreasing HB lifetimes. According to such evidence, one expects a decreasing value of $R$ and thus a larger value of $Y$ for any given value of $R$. Owing to the relevance of the argument, let us derive a quantitative evaluation of $R$ as given by updated predictions about evolutionary times both along the RG and through the HB evolutionary phases. It has been already found that evolutionary times along 

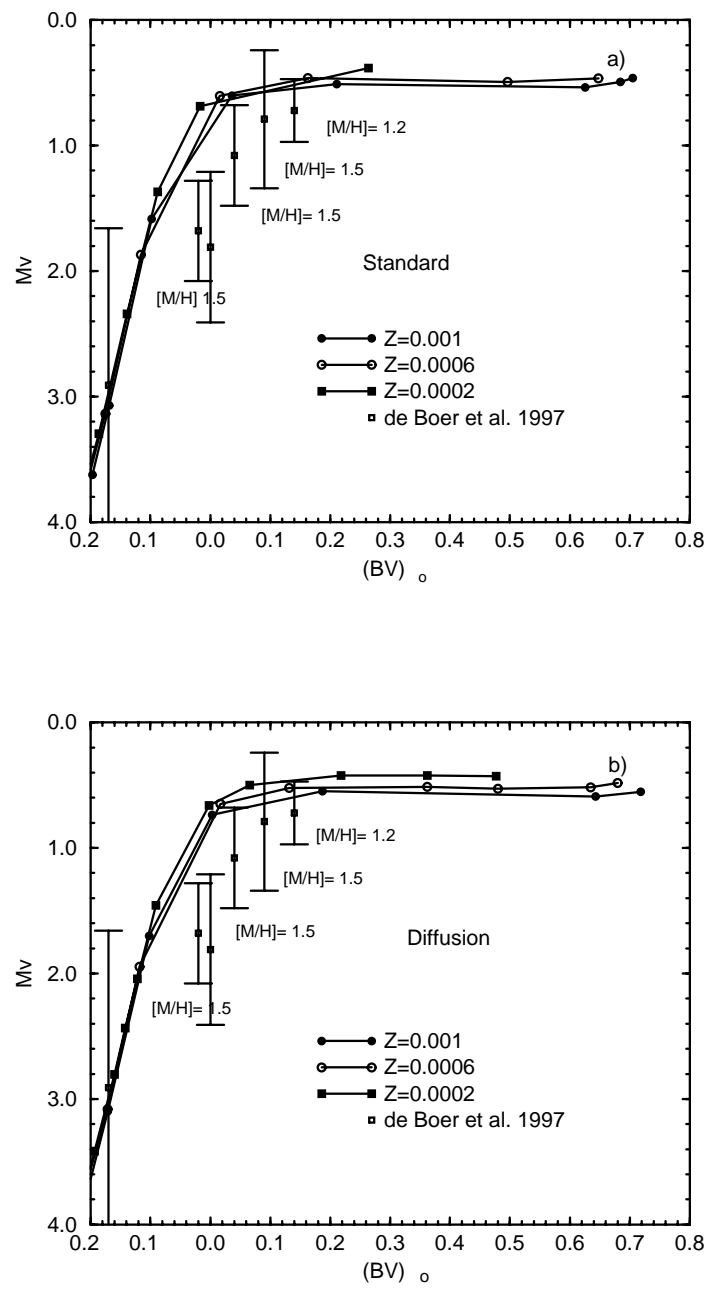

Fig. 10. a) Theoretical ZAHB for standard models compared with Hipparcos estimates of HB magnitudes from De Boer et al. 1997 (see text). When available, labeled metallicities are from Gray et al. 1996. b) As a) but for models computed by accounting for element diffusion

the upper portion of the RG branch show a negligible dependence on both the chemical composition (within Pop. II limits) and mass of the evolving stars (see e.g. Castellani \& Castellani 1993; Bono et al. 1995; Salaris $\&$ Cassisi 1997). Now we find a small but not negligible dependence on the efficiency of sedimentation. By best fitting computational results we find in the interval $1.5 \leq \log L \leq 1.8$ :

\section{No Diffusion}

$$
t^{\text {flash }}-t=730.93-629.14 \log L+144.73(\log L)^{2}
$$

Diffusion

$$
t^{\text {flash }}-t=732.93-625.73 \log L+143.32(\log L)^{2}
$$

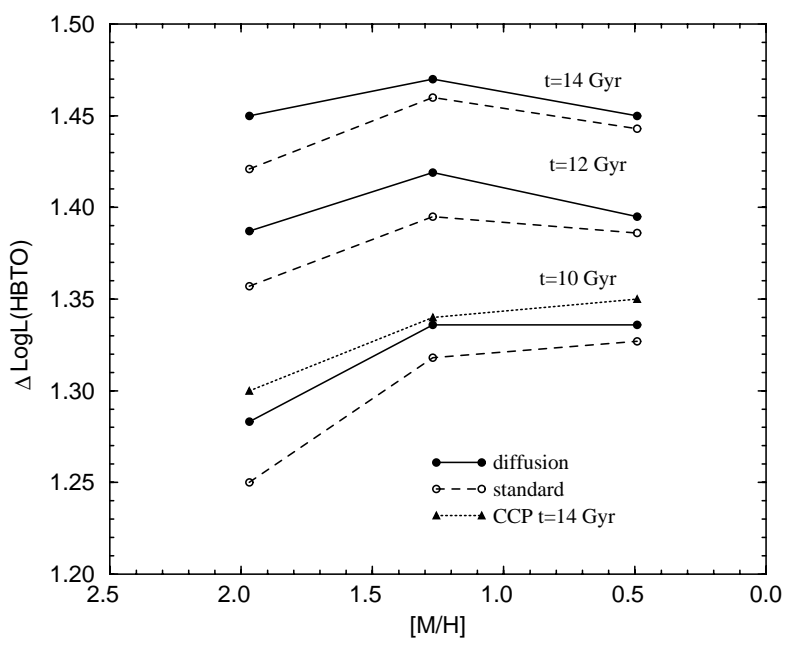

Fig. 11. The dependence on metallicity of the difference in luminosities between ZAHB (at $\log T_{\mathrm{e}}=3.85$ ) and TO $(\Delta \log L(\mathrm{HB}-\mathrm{TO})$ ), as predicted by present models with (solid line) or without diffusion (dashed line) for selected labeled ages. Present results are compared with original predictions in CCP (dotted line)

where $t^{\text {flash }}-t$ represents the time (in $10^{6}$ yr) spent by a RG above the luminosity $L$. However, when $Z=0.006$ these relations can be safely used only in clusters with age lower than, about, 13 Gyr. At larger ages, the clump of stars along the RG branch becomes fainter than the HB luminosity level, as shown by data in the previous Table 8 , and the relations would require a correction to properly account for such an occurrence (see Bono et al. 1995 for a discussion on that matter).

According to the procedure adopted by Bono et al. (1995) we will take as reference the luminosity level of the ZAHB at $\log T_{\mathrm{e}}=3.83$, evaluating the time spent by RG stars above such a luminosity and taking HB evolutionary lifetimes from the models starting $\mathrm{HB}$ evolution at that effective temperature. Table 10 gives data for these two

Table 10. Theoretical estimates of the parameter $R$. Times are in $\mathrm{Myr}$

\begin{tabular}{ccccccc}
\hline \hline$Z$ & 0.0002 & 0.0002 & 0.001 & 0.001 & 0.006 & 0.006 \\
\hline Diffusion & NO & YES & NO & YES & NO & YES \\
\hline & & & & & & \\
$\log L_{\text {ZAHB }} L_{\odot}$ & 1.759 & 1.744 & 1.701 & 1.687 & 1.594 & 1.574 \\
$t_{\text {He }}(\mathrm{Myr})$ & 76.16 & 71.33 & 83.70 & 81.13 & 93.00 & 91.30 \\
$t_{\text {RG }}(\mathrm{Myr})$ & 73. & 77.24 & 79.52 & 85.22 & 68.3 & 83.20 \\
\hline & & & & & & \\
$R(3.83)$ & 1.043 & 0.923 & 1.053 & 0.952 & 1.362 & 1.097 \\
$R(3.83)+0.05$ & 1.142 & 1.010 & 1.395 & 1.034 & 1.525 & 1.203 \\
$R(3.83)+0.10$ & 1.248 & 1.102 & 1.522 & 1.120 & 1.690 & 1.308 \\
\hline \hline
\end{tabular}


ingredients together with the corresponding estimates of $R$ for the labeled choices for the metallicity, with or without allowing for the efficiency of sedimentation. Top to bottom one finds: the luminosity ( $\left.\log L_{\mathrm{ZAHB}}\right)$ of the ZAHB model at $\log T_{\mathrm{e}}=3.83$ the time $\left(t_{\mathrm{He}}\right)$ spent by the same model during the central He burning (until the disappearance of the convective core), the time $\left(t_{\mathrm{RG}}\right)$ spent by RGB stars above $\log L_{\mathrm{ZAHB}}$, the value $(R(3.83))$ of the corresponding $R$ parameter and the same values when the ZAHB luminosity level is artificially increased by $\Delta \log L=0.05$ $(R(3.83)+0.05)$ and $0.1(R(3.83)+0.1)$. As already recognized, one sees that an increase of the metallicity tends to slightly increase the expectations on $R$ for a given value of $Y$. Focusing our attention on the case $Z=0.001$, one finds that when $Y=0.23$ the theoretical prediction given by Bono et al. (1995), $R=1.19$, should now be decreased to $R=1.05$ for the model without sedimentation or to $R=0.95$ if sedimentation is taken into account. According to all available calibrations of $R$ one finds $\delta Y \approx$ $0.4 \delta \log R$. As a consequence, the present evolutionary scenario would predict that our current estimate of original He should be increased by about $\Delta Y \sim 0.02$ if sedimentation is neglected, or by about $\Delta Y \sim 0.04$ with sedimentation at work. As a result, observational data already interpreted in the literature as an evidence for $Y=0.23$ should now lead to the rather unpalatable conclusion $Y \simeq 0.27$.

However, before entering on a discussion of the values in Table 10, one has to note that the calibration of $R$ depends on He-burning evolutionary times which, in turn, are mainly governed by the poorly determined cross section for the ${ }^{12} \mathrm{C}+\alpha$ reaction (see also Dorman 1992). Throughout this paper we adopted for He burning reactions the rates given by Caughlan \& Fowler (1988) which should improve previous evaluations given by the same authors in 1985. Comparison between these two rates shows a rather negligible difference in the triple alpha rate, but a large decrease in the ${ }^{12} \mathrm{C}+\alpha$ rate which, in turn, largely contributes to the decrease of $\mathrm{HB}$ lifetimes one finds in Table 1 between steps 4 and 7 . As a matter of fact, about $60 \%$ of this decrease in HB lifetime (and of the corresponding decrease in the predicted value of $R$ ) can be attributed to these new rates. However, error estimates on such a cross section are still as large as a factor of two, including in this range also previous estimates given by Caughlan et al. (1985). Moreover, numerical experiments performed on HB models adopting recent reaction rates by Buchmann (1996), with error estimates still of about $70 \%$, tend to move the lifetimes toward the values estimated in old computations, based on Caughlan et al. (1985). One can only conclude that theoretical calibrations of $R$ in terms of $Y$ are affected by too large errors to be useful for accurate calibrations of such a relevant parameter, and that the values of $R$ given in Table 10 are still affected by theoretical errors corresponding to an error on $Y$ of about $\Delta Y \sim 0.02-0.03$. If one adds the further errors related to the observational procedure, i.e., the errors on the HB luminosity level, on the bolometric correction for the corresponding RG stars and on the star counts (see, e.g., Brocato et al. 1995) one should conclude that $R$ still appears a too risky parameter to allow evaluations of $Y$ with a reasonable accuracy.

The last two rows in Table 10 finally give theoretical evaluations for $R$ when the adopted luminosity level is artificially increased above the ZAHB level by $\Delta \log L=$ 0.05 and 0.1 , respectively. These values can be used to evaluate theoretical expectations on $R$ when the mean luminosity of RR Lyrae is taken instead of the ZAHB luminosity as reference luminosity level. In the meantime these values give an estimate of the error on $Y$ produced by observational errors at that level. One easily finds that an overestimate by $\Delta \log L=0.05(0.125 \mathrm{mag})$ will produce an overestimate of He by about $\Delta Y \sim 0.015$. Note that previous evaluations of $R$ appear only as a lower limit for theoretical expectations for clusters with a blue HB. Less massive, hot HB structures have He burning evolutionary times increased by $20 \%$ or more (see Fig. 4 and Castellani et al. 1994), with a corresponding increase in the theoretical expectation for the parameter $R$.

\section{Conclusions}

In this paper we have followed the evolution of theoretical predictions concerning Pop. II stellar models vis-a-vis the recent progress in the input physics. Stellar models including all the more recent evaluations of theoretical ingredients have been presented and discussed, with particular regard to the problem of globular cluster ages. We found that these models tend to decrease previous estimates about the cluster age. The account for element sedimentation goes in the same direction. As a whole one finds that "canonical age estimates", as given in CCP, have to be decreased by about $4 \mathrm{Gyr}$, promising a much better agreement with cosmological constraints. We finally drew attention to the large uncertainty of the theoretical procedure adopted to constrain the cluster original abundance of $\mathrm{He}$, concluding that accurate results on that subject must wait for a better determination of the nuclear cross section ${ }^{12} \mathrm{C}+\alpha$. Detailed tabulations on both evolutionary tracks and/or cluster isochrones are available upon request by E-mail.

Acknowledgements. It is a pleasure to thank F. D'Antona for her comments on a preliminary draft of the paper.

\section{References}

Alexander D.R., 1975, ApJS 29, 363

Alexander D.R., Ferguson J.W., 1994, ApJ 437, 879

Bahcall J.N., Pinsonneault M.H., Wasserburg G.J., 1995, Rev. Mod. Phys. 67, 781

Beaudet G., Petrosian V., Salpeter E.E., 1967, ApJ 150, 979 
Bergbusch P.A., VandenBerg D.A., 1992, ApJS 81, 163

Bertelli G., Bressan A., Chiosi C., Fogatti F., Nasi E., 1994, A\&AS 106, 275

Bono G., Castellani V., Degl'Innocenti S., Pulone L., 1995, A\&A 297, 115

Bono G., Caputo F., Cassisi S., Castellani V., Marconi M., 1997a, ApJ 479, 279

Bono G., Caputo F., Cassisi S., Incerpi R., Marconi M., 1997b, ApJ 483, 811

Brocato E., Castellani V., Ripepi V., 1995, AJ 109, 1670

Buchmann L., 1996, ApJ 468, L127

Buzzoni A., FusiPecci F., Buonanno R., Corsi C.E., 1983, A\&A 128, 94

Canuto V.M., Mazzitelli I., 1991, ApJ 370, 295

Caputo F., Martinez Roger C., Paez E., 1987, A\&A 183, 228

Cassisi S., Degl'Innocenti S., Salaris M., 1997, MNRAS 290, 515

Castellani M., Castellani V., 1993, ApJ 407, 649

Castellani V., Degl'Innocenti S., 1995, A\&A 298, 827

Castellani V., Chieffi S., Pulone L., 1991, ApJS 76, 911 (CCP)

Castellani M., Castellani V., Pulone L., Tornambe A., 1994, A\&A 282, 771

Castellani V., Ciacio F., Degl'Innocenti S., Fiorentini G., 1997, A\&A 322, 801 (Paper II)

Caughlan G.R., Fowler W.A., 1988, Atom. Data Nucl. Data Tables 40, 283

Caughlan G.R., Fowler W.A., Harris, M.J., Zimmerman B.A. 1985, Atom. Data Nucl. Data Tables 32, 197 bibitemcha95Chaboyer B., 1995, in "Stellar Evolution: What should be done", Noels A., Fraipont-Caro D., Gabriel M., Grevesse N., Demarque P. (eds.), p. 345

Chaboyer B., Kim Y.-C., 1995, ApJ 454, 767

Chaboyer B., Demarque P., Sarajedini A., 1996a, ApJ 459, 558

Chaboyer B., Demarque P., Kernan P.J., Krauss L.M., Sarajedini A., 1996b, MNRAS 283, 683

Cox A.N., Tabor J.E., 1976, ApJS 31, 271

Dappen W., Mihalas D., Hummer D.G. Mihalas B.W., 1988, ApJ 332, 261

D'Antona F., Caloi V., Mazzitelli I., 1997, ApJ 477, 519

De Boer K.S., Tucholke H.-J., Schmidt J.H.K., 1997, A\&A 317, L23

DeWitt H.E., Graboske H.C., Cooper M.S., 1973, ApJ 181, 439

Dorman B., 1992, ApJS 81, 221

Dorman B., VandenBerg D.A., 1989, ApJ 343, 750

Dorman B., Rood R.T., O' Connell R.W., 1993, ApJ 419, 596

Eggleton P.P., Faulkner J., Flannery B.F., 1973, A\&A 23, 325

Fowler W.A., Caughlan G.R., Zimmerman B.A., 1975 , ARA\&A 13, 69

Graboske H.C., DeWitt H.E., Grossman A.S., Cooper M.S., 1973, ApJ 181, 457

Grevesse N., Noels A., 1993, in "Origin and Evolution of the elements", Prantzos N., Vangioni-Flam E., Casse M̀. (eds.). Cambridge Univ. Press, Cambridge, p. 15

Gray R.D., Corbally C.J., Davis Philip A.G., 1996, AJ 112, 2291

Gross P.G., 1973, MNRAS 164, 65
Haft M., Raffelt G., Weiss A., 1994, ApJ 425, 222

Harris, M.J., Fowler W.A.,Caughlan G.R., Zimmerman B.A., 1983, ARA\&A 17, 241

Huebner W.F., Merts, A.L., Magee N.H., Argo M.F., 1977, Los Alamos Sci. Lab. Rept. (LA-6760-M)

Iben I. Jr., 1968, Nat 220, 143

Iglesias C.A., Rogers F.J., 1996, ApJ 464, 943

Itoh N., Totsuji H., Ichimaru S., 1977, ApJ 218, 477

Itoh N., Totsuji H., Ichimaru S., DeWitt H.E., 1979, ApJ 234, 1079

Itoh N., Mitake S., Iyetomi H., Ichimaru S., 1983, ApJ 273, 774

Itoh N., Adachi T., Nakagawa M., Kohyama Y., 1989, ApJ 339, 354

Itoh N., Adachi T., Nishikawa A., Kohyama Y., 1996, ApJ 470, 1015

Kurucz R.L., 1992, in IAU Symp. 149, The Stellar Populations of Galaxies, Barbuy B., Renzini A. (eds.). Dordrecht, Kluwer, p. 225

Lee Y.-W., Demarque P., 1990, ApJS 73, 709

Mazzitelli I., D'Antona F., Caloi V., 1995, A\&A 302, 382

Mihalas D., Hummer D.G., Mihalas B.W., Däppen W., 1990, ApJ 360, 300

Mitler H.E., 1977, ApJ 212, 513

Munakata H., Kohyama Y., Itoh N., 1985, ApJ 296, 197

Proffitt C.R., VandenBerg D.A., 1991, ApJS 77, 473

Richarson M.B., Van Horn M.H., Ratcliff K.F., Malone R.C., 1982, ApJ 255, 624

Richer H.B., Fahlman G.F., VandenBerg D.A., 1988, ApJ 329, 187

Rogers F.J., 1994, in IUA Colloq. 147, in Chabrier G., Schatzman E.L. (eds.), "The equation of state in astrophysics". Cambridge University Press, Cambridge, p. 16

Rogers F.J., Iglesias C.A., 1992, ApJS 79, 507

Rogers F.J., Swenson F.J., Iglesias C.A., 1996, ApJ 456, 902

Salaris M., Cassisi S., 1997, MNRAS 289, 406

Salaris M., Chieffi S., Straniero O., 1993, ApJ 414, 580

Salaris M., Degl'Innocenti S., Weiss A., 1997, ApJ 479, 665 (Paper I)

Seaton M.J., Yu Yan, Mihalas D., Pradhan A.K., 1994, MNRAS 266, 805

Straniero O., 1988, A\&AS 76, 157

Straniero O., Chieffi A., 1991, ApJS 76, 525

Sweigart A.V., 1987, ApJS 65, 95

Sweigart A.V., Gross P.G., 1978, ApJS 36, 405

Tanvir N.R., Shanks T., Ferguson H.C., Robenson D.R.T., 1995, Nat 377, 27

Thoul A.A., Bahcall J.N., Loeb A., 1994, ApJ 421, 828

VandenBerg D.A., 1992, ApJ 391, 685

VandenBerg D.A., Bolte M., Stetson P.B., 1996, ARA\&A 34, 461

VandenBerg D.A., Irwin A.W., 1997, in "Advances in Stellar Evolution", Rood R.T., Renzini A. (eds.), CUP, p. 22

Van Den Bergh S., 1994, PASP 106, 1113

Weiss A., Peletier R., Matteucci F., 1995, A\&A 296, 73 